\author{
J. Bösel ${ }^{1}$, P. Berlit ${ }^{2}$ \\ ${ }^{1}$ Klinik für Neurologie, Klinikum Kassel, Kassel, Deutschland \\ ${ }^{2}$ Deutsche Gesellschaft für Neurologie, Berlin, Deutschland
}

\title{
Neurologische Auswirkungen von COVID-19
}

\section{Hintergrund}

Die Pandemie durch das Coronavirus SARS-CoV-2 („severe acute respiratory syndrome coronavirus 2") hat seit Dezember 2019 die Welt und die Medizin im Griff. Zum Zeitpunkt der Verfassung dieses Artikels sind weltweit über 3 Mio. Infektionen bestätigt und über 220.000 Menschen an der Infektion verstorben. Während diese so schon schockierenden Zahlen ganz sicher aufgrund der regional sehr unterschiedlichen Test- und Dokumentationsaktivität nicht zuverlässig sind, sondern tatsächlich deutlich höher liegen dürften, mag die Situation in Deutschland etwas zuverlässiger erfasst sein: aktuell fast 160.000 Infizierte und über 6000 Verstorbene [1]. Patienten mit milden oder schweren Verläufen der durch SARS-CoV-2 verursachten Erkrankung COVID-19 belegen zunehmend auch deutsche Kliniken, mit Hotspots im Süden und Nordwesten, was zu drastischen Umstrukturierungen und Prozessänderungen in den Krankenhäusern führte. Natürlich ist dies in erster Linie die Stunde der Virologen, Pneumologen und Intensivmediziner, aber auch Neurologen müssen sich aus verschiedenen Gründen ganz erheblich mit dieser neuen Viruserkrankung befassen. Neben indirekten Auswirkungen, die die Pandemie im Sinne von „Kollateralschäden“ auf die Versorgung neurologischer Patienten hat, mehren sich die Hinweise darauf, dass das Virus das Nervensystem selbst befällt und bei einem Teil der Patienten genuine neurologische Erkrankungen (sog. NeuroCOVID) oder zumindest Komplikationen im COVID-19-Verlauf hervorruft.

\section{Indirekte Auswirkungen der Pandemie auf die Versorgung neurologischer Patienten}

\section{Einschränkung der (elektiven) neurologischen Versorgung in Praxen und Kliniken}

Die Einschränkungen im öffentlichen Leben zur Bremsung bzw. Verhinderung der Transmission führten in den letzten Wochen dazu, dass in neurologischen Praxen, Ambulanzen und Kliniken viele elektive Patientenkontakte eingestellt oder stark eingeschränkt wurden. Obwohl diese Maßnahmen zweifellos notwendig sind, birgt dieses Vorgehen zahlreiche Risiken: Behandlungspfade und -verhältnisse werden beeinträchtigt, chronische Erkrankungen können bei suboptimaler Behandlung auf ein kritisches Niveau voranschreiten, Komplikationen werden womöglich übersehen. Hinzu kommen ein vermutlich erheblicher

\section{Redaktion}

P. Berlit, Berlin

DGNeurologie 2020 · 3 (4): 277-284

https://doi.org/10.1007/s42451-020-00191-9

Online publiziert: 11 . Mai 2020

(c) Springer Medizin Verlag GmbH, ein Teil von Springer Nature 2020
Stau und dann Ansturm nach Wiedereröffnung und die Verunsicherung der Patienten. Die aktive sektorenübergreifende Kommunikation zwischen Klinikärzten, Rehabilitationsmedizinern und Niedergelassenen, Ausnahmeregelungen für zeitrelevante Diagnostik und Therapie unter Schutzmaßnahmen und die Einrichtung von Videosprechstunden sind hier sinnvolle Alternativen, die auch schon vielerorts von Neurologen etabliert wurden. Weltweit hat COVID-19 z. B. die Teleneurologie katalysiert, was sicherlich auch für die Zeit nach der Krise vorteilhaft ist [2].

\section{Umgang mit immunsupprimierten/-modulierten Patienten}

Schon früh nach Beginn der Pandemie trat die Frage auf, wie es sich mit dem Risiko bei immunsupprimierten und/oder immunmodulatorisch behandelten neurologischen Patienten verhält, also z. B. solchen mit multipler Sklerose, chronischer inflammatorischer demyelinisierender Polyneuropathie, multifokaler motorischer Neuropathie, Myasthenia gravis oder Hirntumoren. Aber auch Patienten mit neurodegenerativen und neuromuskulären Erkrankungen sind aus verschiedenen Gründen eine Risikogruppe. Schnell gaben internationale und nationale Fachgesellschaften zu diesen und anderen neurologischen Erkrankungen Empfehlungen heraus, mit zahlreichen wichtigen Hinweisen zur Prävention und zum Monitoring und mit - sehr vereinfacht zusammengefasst - dem Tenor, immunmodulatorische Therapien, die für den Krankheitsverlauf wichtig sind, nicht wegen der Pandemie zu pausieren oder abzubrechen. Diese Empfehlungen sind über die Homepage der DGN abrufbar [3].

\section{Dynamik bei Schlaganfällen und anderen neurologischen Notfällen}

Noch kritischer jedoch sind vermutlich pandemieassoziierte Veränderungen bei der Vorstellung und Zuweisung von Patienten mit neurologischen Notfällen bzw. alarmierenden Symptomen in den Notaufnahmen oder den Praxen. Wie auch von anderen Fachbereichen beobachtet, waren diese in den letzten Wochen - z. T. stark - rückläufig. Weil kaum angenommen werden kann, dass sich unter der Pandemie tatsächlich Inzidenzen verändern, muss befürchtet werden, dass aus Sorge vor einer Infektion mit SARS-CoV-2 oder wegen der Aufmerksamkeit, die COVID-19 berechtigterweise erfährt, Patienten mit neurologischen Notfallerkrankungen ihre Symptome „aussitzen" bzw. nicht wahrgenommen oder zugewiesen werden. Auch Auswirkungen der Pandemie auf die präklinische Logistik, Ressourcenverfügbarkeit oder Barrieremaßnahmen können hier eine große Rolle spielen. Die Vorstellungen von Patienten mit Schlaganfällen, besonders leicht- und mittelgradig Betroffener, sind klar rückläufig [4], obwohl von manchen Autoren ein schlaganfallförderndes Potenzial von COVID-19 postuliert 
wird (s. unten). In diversen Medien riefen Vertreter der DGN bereits eindringlich die Öffentlichkeit und Rettungsdienste dazu auf, Schlaganfallpatienten nicht zu vernachlässigen und diese schnellstmöglich klinisch vorzustellen, u.a. um ihnen eine rekanalisierende Therapie zukommen zu lassen. Diesem Beispiel sollten Schlaganfallneurologen allerorts folgen, insbesondere neurovaskuläre Netzwerke können hierfür gute Dienste leisten. Die Akutbehandlung bei Schlaganfall in den Kliniken muss unter den derzeit gebotenen Schutzmaßnahmen erfolgen [5]. Dies gilt insbesondere für die Thrombektomie in der $\mathrm{Zu}-$ sammenarbeit von Neurologen, interventionellen Neuroradiologen, Anästhesisten und Pflegekräften wegen der Nähe zum Patienten und der Gefahr der Aerosolverbreitung. Mehrere mit diesem Setting betraute Fachgesellschaften gaben hierzu Empfehlungen heraus. Hierzu zählen die Einordnung jedes Patienten als prinzipiell COVID-19-verdächtig mit der Notwendigkeit einer umgehenden Testung, die Bevorzugung einer Intubationsnarkose (zur Vorbeugung einer möglichen unkontrollierten Notfallintubation während der Intervention), die videolaryngoskopische Intubation in Räumlichkeiten mit Absaugung, die Reduktion der Beteiligten auf die wirklich notwendige Anzahl und die Verwendung von persönlichem Schutz- und Barrierematerial für Patient und Behandler [6].

\section{Triage für Ressourcenallokation neurologischer Patienten}

Obwohl die Situation an deutschen Kliniken - wenn auch regional sehr unterschiedlich - momentan kontrolliert zu sein scheint und uns hoffentlich Katastrophenszenarien wie in Italien erspart bleiben [7], kann es dennoch sein, dass die Allokation von Ressourcen, insbesondere Intensivbetten, bei uns irgendwann triagiert werden muss. Die DIVI (Deutsche Interdisziplinäre Vereinigung für Intensiv- und Notfallmedizin) veröffentlichte für alle Fachbereiche hierzu eine S1-Leitlinie [8]. Es geht darin u.a. um die Frage der Aufnahme auf die Intensivstation zur Beatmung oder sogar den Abbruch einer begonnenen Intensivtherapie, weil das Bett für eine aussichtsreichere Behandlung benötigt wird. Sowohl die Abwägung zwischen einem COVID-19- und einem Nicht-COVID-19-Patienten als auch die Abwägung zwischen Nicht-COVID-19-Patienten, weil in einer Klinik wegen COVID-19-bedingter Verschiebung von Intensivressourcen der Platz für andere intensivpflichtige Patienten knapper geworden ist, bedeutet in jedem Fall ein drastisches ethisches Dilemma, das den einzelnen extrem belastet. Ein gemeinsames Vorgehen ist erforderlich, um diese Belastung erträglicher zu machen und so fair wie möglich zu entscheiden. Neurologen und Neuro-Intensivmediziner müssen sich dann in lokale multidisziplinäre Prognose- und Allokationsteams einbringen, denn für die Beurteilung einer intensivstationären Behandlungsaussicht für Patienten mit fortgeschrittenen neurodegenerativen oder aber auch schweren akutneurologischen Erkrankungen ist unbedingt neurologische Expertise erforderlich [9]. Dies ergab auch eine aktuelle Umfragestudie der DGNIForschungsgruppe IGNITE.
Direkte Auswirkungen von SARS-CoV-2 auf das

Nervensystem - Neuro-COVID

\section{Berichte zu Kohorten mit neurologischen Manifestationen}

Nicht lange nach den ersten Publikationen zu den klinischen Charakteristika von COVID-19-Kohorten mit internistischem Fokus aus China tauchten auch Beschreibungen von Patientengruppen mit neurologischen Manifestationen auf. Die initial größte Arbeit kam Ende Februar von der Gruppe um Bo $\mathrm{Hu}$ aus 3 designierten COVID-19-Zentren in Wuhan, China, und beschrieb retrospektiv unter 214 konsekutiven Patienten 78 Kranke (36,4\%) mit neurologischen Manifestationen, wobei die Autoren solche des ZNS, des PNS und der Skelettmuskulatur unterschieden [10]. Danach folgten mit erstaunlicher Dynamik weitere Fallberichte, Fallserien, Studien und sogar erste Reviews zur Beteiligung des Nervensystems zunächst in PräPrint-Medien, bald darauf in namhaften Journalen wie Lancet, JAMA oder NEJM. Das Interesse der neurologischen Gemeinschaft ist somit sicher geweckt, auch wenn noch viel Unklarheit darüber besteht, was an den Beobachtungen eine spezifische Beteiligung des Nervensystems, was eine unspezifische Auswirkung der systemischen Erkrankung auf das Nervensystem und was eher zufällige Begleiterscheinungen sind Eine Auswahl von Publikationen zu Neuro-COVID findet sich in Tab. 1.

\section{Symptome}

Während die häufigsten Symptome, mit denen COVID-19Patienten sich präsentieren, Fieber (um 90\%) und Husten (um 70\%) sind [19], wurden in Charakterisierungen allgemeiner Kohorten aus China auch Symptome gefunden, die einen gewissen, wenn auch evtl. eher unspezifischen, Bezug zum Nervensystem haben, wie Kopfschmerzen (14-43\%), Muskelschmerz (15-22\%), Fatigue (23-73\%) oder Übelkeit und Erbrechen (4-9\%; [19-22]). Eine wegen ARDS beatmungspflichtige Kohorte der Universitätsklinik Aachen bot hinsichtlich solcher Symptome Häufigkeiten für Kopfschmerz von nur $2 \%$, Muskelschmerz von $12 \%$, Müdigkeit von $12 \%$, Übelkeit und Erbrechen von 2-4\% [23]. Studien, in denen gezielt nach neurologischen Symptomen gefahndet wurde, beschrieben bei der Aufnahme oder im Verlauf (plötzliche) Geruch- und Geschmackstörungen (10-70\%), Kopfschmerzen $(13 \%)$, Schwindel (17\%), Halluzinationen, Verwirrtheit, Dysexekutivstörungen (nach Intensivverlauf 36\%), Agitation (im Intensivverlauf 69\%) Vigilanzminderung (8-15\%), Neuralgie (2\%), epileptische Anfälle (1\%), Ataxie (1\%), plötzliche neurologische Defizite (3\%), oder Pyramidenbahnzeichen (im Intensivverlauf $67 \%)$; [11, 23, 24]. Eine Zusammenfassung der bisher berichteten neurologischen Symptome bietet Tab. 2 .

\section{Komplikationen und Erkrankungen}

Bald darauf wurden korrelierend mit solchen Symptomen oder auch unabhängig davon in Fallberichten oder Fallserien Patientenverläufe mit Befall oder Beeinträchtigung des Nervensystems beschrieben. In einer Serie aus Strasbourg berichteten Helms et al. [11] bei 58 Patienten mit schweren Verläufen (ARDS und Intensivbehandlung) unterschiedliche ZNS-Manifestationen, großteils während der Unterbrechung der Analgosedierung, wie Delir und klinische Hinweise auf Pyramiden- 
Hier steht eine Anzeige.

Springer 
Tab. 1 Ausgewählte Studien und Berichte (alle aus 2020, alle zu COVID-19-Patienten)

\begin{tabular}{|c|c|c|}
\hline Autor/Land & Methode & Hauptergebnisse \\
\hline $\begin{array}{l}\text { Mao et al. [10]/ } \\
\text { China }\end{array}$ & $\begin{array}{l}\text { Retrospektive Serie von } 214 \\
\text { konsekutiven Patienten }\end{array}$ & $\begin{array}{l}\text { ZNS-Symptome bei } 25 \% \text {, darunter Kopfschmerz (13\%), Schwindel (17\%), plötzliches Defizit (3\%), } \\
\text { Ataxie }(0,5 \%) \text {, Anfälle }(0,5 \%)\end{array}$ \\
\hline $\begin{array}{l}\text { Helms et al. [11]/ } \\
\text { Frankreich }\end{array}$ & $\begin{array}{l}\text { Retrospektiven Serie von } \\
58 \text { konsekutiven ICU-Pati- } \\
\text { enten }\end{array}$ & $\begin{array}{l}\text { ZNS-Symptome zumeist nach Beendigung der Analgosedierung bei } 84 \% \text {, darunter Agitation } \\
(69 \%) \text {, Pyramidenbahnzeichen ( } 67 \%) \text {, dysexekutives Syndrom ( } 36 \%) \text {; meningeales Enhancement } \\
\text { und gestörte Perfusion in manchen MRT, kein Virusnachweis im Liquor }\end{array}$ \\
\hline Li [12]/China & $\begin{array}{l}\text { Retrospektive Serie von } 221 \\
\text { konsekutiven Patienten }\end{array}$ & $\begin{array}{l}\text { Neue zerebrovaskuläre Ereignisse bei } 11 \text { Patienten, darunter Hirninfarkt }(5 \%) \text {, intrazerebrale Blu- } \\
\text { tung }(0,5 \%) \text {, Hirnvenen- und Sinusthrombose }(0,5 \%) \text {; hyperkoaguable und inflammatorische La- } \\
\text { borkonstellation }\end{array}$ \\
\hline $\begin{array}{l}\text { Zhang et al. [13]/ } \\
\text { China }\end{array}$ & Fallserie von 3 Patienten & Multiple Hirninfarkte, Antiphospholipidantikörper \\
\hline $\begin{array}{l}\text { Oxley et al. } \\
\text { [14]/USA }\end{array}$ & Fallserie von 5 Patienten & Großgefäßverschlusshirninfarkte („large vessel occlusion“) bei Alter $<50$ Jahre \\
\hline $\begin{array}{l}\text { Poyiadji et al. } \\
{[15] / \text { USA }}\end{array}$ & Fall einer Patientin & $\begin{array}{l}\text { Akute hämorrhagische nekrotisierende Enzephalopathie mit bilateralen mesiotemporalen und } \\
\text { thalamischen MRT-Läsionen }\end{array}$ \\
\hline $\begin{array}{l}\text { Moriguchi et al. } \\
{[16] / J a p a n}\end{array}$ & Fall eines Patienten & $\begin{array}{l}\text { Erster (?) publizierter Bericht einer Meningoenzephalitis mit Anfallsserie und Virusnachweis im } \\
\text { Liquor }\end{array}$ \\
\hline $\begin{array}{l}\text { Paniz-Mondolfi et } \\
\text { al. [17]/USA }\end{array}$ & Fall eines Patienten & $\begin{array}{l}\text { Erster (?) publizierter Bericht einer fatalen Enzephalitis mit autoptischem Nachweis von Virus in } \\
\text { Neuronen und kapillarem Endothel des Frontalhirns }\end{array}$ \\
\hline $\begin{array}{l}\text { Toscano et al. [18]/ } \\
\text { Italien }\end{array}$ & Fallserie von 5 Patienten & $\begin{array}{l}\text { Guillain-Barré-Syndrom 5-10 Tage nach ersten COVID-19-Symptomen, kein Virusnachweis im } \\
\text { Liquor }\end{array}$ \\
\hline
\end{tabular}

Tab. 2 Neurologische Symptome im Zusammenhang mit
COVID-19
Riechstörung
Geschmackstörung
Kopfschmerz
Sog. Muskelschmerz
Sog. Nervenschmerz
Schwindel
Übelkeit
Erbrechen
Halluzinationen
Orientierungsstörung, Verwirrtheit
Vigilanzstörung
Anfälle
Ataxie
Meningismus

bahnaffektionen oder kortikale Dysfunktionen. Paraklinisch zeigten 8 dieser Patienten eine diffuse Hirnfunktionsstörung im EEG, von 13 Patienten mit MRT 11 Perfusionsstörungen (3-mal mit akuter zerebraler Ischämie) und 8 ein leptomeningeales Enhancement. Die Liquoranalyse bei 7 Patienten war ohne Virusnachweis [11]. Die Autoren waren sich unsicher, ob die neurologischen Symptome spezifische Folgen der Virusinfektion, einer übermäßigen Zytokinausschüttung oder der Intensivtherapie sind. Ein Zytokinsturm mag dem eindrucksvollen Fallbericht einer COVID-19-assoziierten akuten hämorrhagisch-nekrotisierenden Enzephalopathie zugrunde gelegen habe, die Poyiadji et al. [15] bei einer Ende 50-jährigen Patientin in Detroit fanden. Nach 3 Tagen Fieber, Husten und Verwirrtheit kam es zu einem schweren respiratorischen Versagen unter der Diagnose von COVID-19. Die CT zeigte bitha- lamische Hypodensitäten, die MRT bilaterale mesiotemporale und ausgeprägte thalamische Hyperintensitäten in der FLAIRWichtung, thalamische Hämorrhagien in der Suszeptibilitätswichtung sowie ein Ringenhancement nach KM-Gabe [15]. Der Beweis des direkten Befalls des ZNS wurde bei einem japanischen Patienten mit Meningoenzephalitis geführt, bei dem nach einem fieberhaften Verlauf mit Fatigue über wenige Tage epileptische Anfälle aufgetreten waren. Der Patient entwickelte im Verlauf eine schwere Pneumonie. Die Liquoranalyse zeigte 12 Zellen/ $\mu$ l und erbrachte den Nachweis von SARS-CoV-2, während ein Rachenabstrich negativ ausgefallen war. In der MRT boten sich DWI-Hyperintensitäten der Ventrikelwand, FLAIR-Hyperintensitäten mesiotemporal und hippocampal [16]. Ein weiterer Fall mit Meningoenzephalitis und Virusnachweis im Liquor soll im „Beijing Ditan Hospital“, China, gelungen sein [25]. Am „Mount Sinai Hospital“, New York, erfolgte der autoptische Nachweis von SARS-CoV-2 im Frontallappen einer 74-jährigen Parkinsonpatientin, die mit Zunahme von Tremor und Gangstörung, Fieber sowie Verwirrtheit vorgestellt wurde und bei COVID-19-Diagnose innerhalb von 11 Tagen nach schwerem respiratorischem Verlauf verstorben war. Die neuropathologische Aufarbeitung zeigte nicht nur Viruspartikel in den Vakuolen von Neuronen, sondern auch Vesikel mit Virusmaterial in benachbarten Endothelzellen als möglichen Hinweis auf eine hämatogene Einwanderung [17]. Weitere Fallberichte zu Patienten mit enzephalitisch bzw. enzephalopathisch zu vermutenden Verläufen, also mit Symptomen wie Orientierungsstörungen, Halluzinationen, Verwirrtheit, Anfällen oder Vigilanzminderungen, mitunter ohne sonstige typische COVID-19-Symptome, liegen vor und werden weiter publiziert.

Eine Reihe von Fallserien und eine retrospektive Beobachtungsstudie existieren auch zu COVID-19-Patienten mit zerebrovaskulären Erkrankungen [26]. Li et al. stellten unter 


Tab. 3 Neurologische Manifestationen im Zusammenhang mit
COVID-19
Meningoenzephalitis
Enzephalopathie (,toxisch“, hypoxisch)
Akute hämorrhagische nekrotisierende Enzephalopathie
Akuter ischämischer Schlaganfall
Intrazerebrale Blutung
Hirnvenen- und Sinusthrombose
Epileptische Anfälle
Guillain-Barré-Syndrom
Miller-Fisher-Syndrom
(Myopathie, Rhabdomyolyse)

221 konsekutiven COVID-19-Patienten des „Wuhan Union Hospital“, Wuhan, China, bei 11 (5\%) AIS (akuter ischämischer Schlaganfall), $1(0,5 \%)$ ICB und bei $1(0,5 \%)$ eine CVST (zerebrale Venen- und Sinusthrombose) fest. Zwar waren die Patienten mit diesen vaskulären Ereignissen älter und durch mehr vaskuläre Risikofaktoren belastet als die COVID-19Patienten ohne Schlaganfälle, aber sie hatten auch deutlich mehr Laborveränderungen, die Inflammation und Hyperkoagulabilität reflektierten [12]. Bei einer Serie von 3 weiteren COVID-19-positiven Patienten aus derselben Klinik fanden sich multiple Hirninfarkte (manche mit zusätzlichen Infarkten in anderen Körperregionen) bei laborchemischen Hinweisen auf eine erhöhte Thromboseneigung mit Leukozytose, Fibrinogenämie, D-Dimer und Antiphospholipidantikörpern [13]. In einer holländischen Studie von 184 intensivpflichtigen COVID19-Pneumonien fanden sich in $31 \%$ thrombotische Komplikationen, v. a. Beinvenenthrombosen und Lungenembolien, trotz routinemäßiger Heparingabe [27]. Kürzlich fielen in den USA v. a. Verschlüsse von großen Hirngefäßen bei Patienten unter 50 Jahren ohne sonstige Schlaganfallrisikofaktoren auf [14]. Andererseits legen Nachrichten aus Krisenherden in Asien oder Italien ein erhöhtes Auftreten von intrakranialen Blutungen nahe.

Im Zusammenhang mit COVID-19 wurden klinisch und elektrophysiologisch sowohl ein Guillain-Barré-Syndrom (GBS) als auch die Miller-Fisher-Variante (MFS) beschrieben und als para- bzw. postinfektiös eingeordnet; bei zytoalbuminärer Dissoziation im Liquor gelang ein Virusnachweis nicht [18, 28]. Gangliosidantikörper wurden nur beim MFS, nicht beim GBS gefunden. Auffällig war in den berichteten Fällen die sehr kurze Latenz zwischen COVID-19-Pneumonie und neurologischer Folgeerkrankung, sodass eine Begleitpolyneuritis bzw. eine „critical illness neuropathy“ abzugrenzen sind. Die Gabe von IVIG scheint wirksam zu sein. Ob der therapeutische Einsatz von Antikörpern aus dem Blut rekonvaleszenter Patienten auch ein GBS auszulösen vermag, bleibt abzuwarten. In Tab. 3 sind bislang berichtete neurologische Erkrankungen zusammengefasst.

\section{Pathophysiologie}

Der Neurotropismus von Coronaviren ist seit Jahrzehnten bekannt und wurde nicht nur in Zellkulturen und Tierexperimenten, sondern auch während der SARS- und MERS-Epidemien an Menschen klinisch, liquordiagnostisch und autoptisch nach- gewiesen [29]. Diese Viren sind dort in der Lage, neuronale Funktionsstörungen, Inflammation oder Degeneration hervorzurufen oder auch nur zu residieren. Das Genom und der zelluläre Invasionsmechanismus des COVID-19 verursachenden SARS-CoV-2 sind entschlüsselt und ähneln dem von SARS$\mathrm{CoV}[30,31]$ : Wie dieses dockt SARS-CoV-2 mit seinem Spikeprotein $S$ an die Zielzelle über das Angiotensinkonversionsenzym 2 (ACE2) als Rezeptor an und wird, vermittelt durch die „transmembrane protease serine 2“ (TMPRSS2), endozytiert, sodass hier ein vielversprechender pharmakologischer Ansatzpunkt durch Blocker bzw. Antikörper aus dem Blut rekonvaleszenter Patienten gesehen wird [32]. Die Unterschiede in der Genetik und Expression von ACE2 werden von manchen Autoren als wichtige Faktoren für die regionale und altersbezogene Verteilung der Pandemie angesehen. Die Affinität zu ACE2 ist für SARS-CoV-2 scheinbar 10- bis 20-mal höher als bei SARS$\mathrm{CoV}$, sodass die ACE2-Expression verschiedener Zelltypen entscheidend für die Organausbreitung des Virus sein dürfte. Neben respiratorischen Zellen wird ACE2 von vielen anderen Zellen exprimiert, so auch von Skelettmuskelzellen, Endothel- und (Riech-)Epithelzellen, Gliazellen und Neuronen [33]. Das Enzym ACE2 ist Andockstelle für das Virus, es gilt aber auch als neuroprotektiver Gegenspieler des ACE, für das im Gehirn die Vermittlung von oxidativem Stress, Inflammation, Apoptose und Degeneration beschrieben wurde. Die Inaktivierung von ACE2 durch Virusbindung könnte so möglicherweise diesen Prozessen Vorschub leisten. Womöglich werden durch eine Beeinträchtigung des Renin-Angiotensin-Systems auch die zerebrale Neurotransmitterregulation und die Bildung von Azetylcholin, Serotonin und Dopamin gestört. Schließlich kann ein Herunterregulieren von ACE2 durch verstärkte Bindung eine schwer steuerbare Hypertension auslösen, mit denkbaren Folgen wie Hirnödem oder Hirnblutungen.

Für die Invasion von SARS-CoV-2 ins ZNS werden im Wesentlichen 2 Routen diskutiert:

Ein Weg ist die hämatogene Ausbreitung über eine ACE2vermittelte Transmission durch Endothelzellen bzw. die virusassoziierte Schädigung der Blut-Hirn-Schranke, wobei die bei COVID-19 beobachtete trägere Mikrozirkulation durch eine längere Kontaktzeit mit dem Endothel eine begünstigende Rolle spielen könnte. Für diese Route gibt es zumindest elektronenmikroskopische Hinweise aus einer Autopsie [17]. Ferner werden die Verbreitung mittels Blutzellen als Trojaner und die lymphatische Ausbreitung als Invasionswege diskutiert.

Eine andere Route ist die transneuronale, also transsynaptische antegrade oder retrograde Verbreitung über Nerven, insbesondere Hirnnerven. Analog zu anderen Coronaviren und angesichts des initialen Befalls der oberen und unteren Atemwege stehen hier insbesondere die Hirnnerven I, VII, IX und X im Verdacht. Die Hirnnerven als Invasionsweg führten zu der Überlegungen, dass so der Hirnstamm und damit Atemwegsregulationszentren beeinträchtigt werden und $\mathrm{zu}$ Atemversagen beitragen könnten [34]. Obwohl tatsächlich Fälle von Atemversagen „ohne Dyspnoe“ und unklare häusliche Todesfälle, scheinbar ohne klassische COVID-19-Symptome, beschrieben wurden, gibt es hierfür bislang wenig belastbare Daten. Die meisten Patienten entwickeln eine schwere Pneumonie, ARDS und ein Typ-1-Atemversagen (Oxygenierungsstörung). Für die Bestätigung der oben genannten Theorie wären ein Typ-2- 


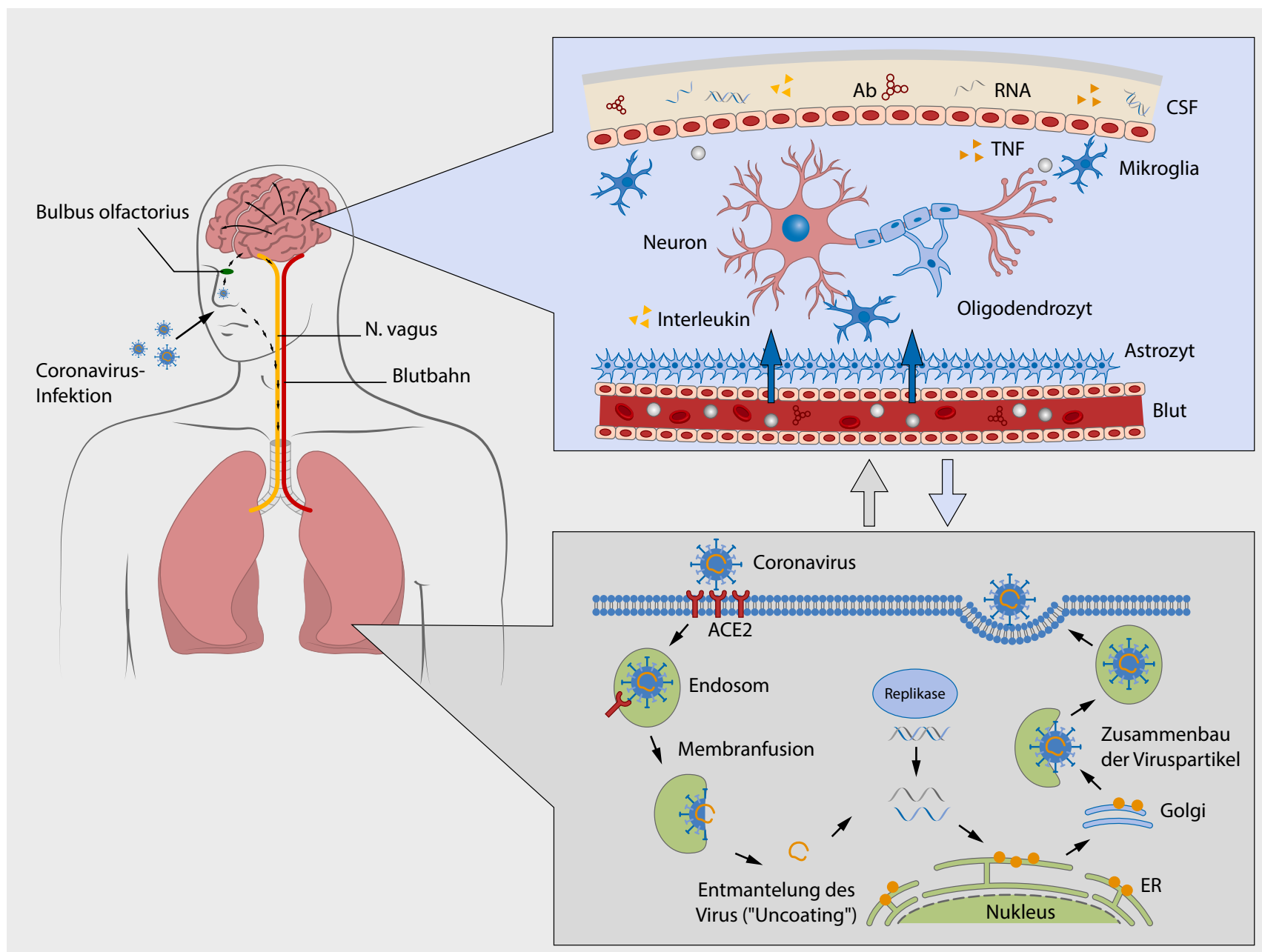

Abb. 1 Mögliche Wege der Neuroinvasion und Pathophysiologie von SARS-CoV-2 („severe acute respiratory syndrome coronavirus 2“), Ab Antikörper, ACE2 Angiotensinkonversionsenzym 2, CSF Liquor cerebrospinalis, ER endoplasmatisches Retikulum, RNA Ribonukleinsäure, TNF Tumor-Nekrose-Faktor. (Adaptiert nach [36])

Atemversagen (Ventilationsstörung), wenig Pneumonie und idealerweise der Virusnachweis im Liquor sowie MRT-Zeichen einer Affektion von Hirnstamm und/oder Medulla oblongata $\mathrm{zu}$ fordern [35]. Viel Aufmerksamkeit und Spekulation erfuhren Riechstörungen als Symptom, auch weil in diesen ein starker Hinweis auf eine Beteiligung des Nervensystems und eine transnasale Invasion ins ZNS gesehen wurde [36]. In darauf fokussierten Studien wurden Anosmie und Ageusie als (teils isolierte) Symptome von COVID-19 mit Häufigkeiten von $70-80 \%$ der sicherlich selektierten Populationen beschrieben, waren dabei aber oft transient und mit milden Verläufen verbunden [24, 37]. Die Route über infiziertes Riechepithel, olfaktorische Neuronen durch die Lamina cribrosa zum Bulbus olfactorius und von dort aus in anderen Hirnregionen wirkt in der Tat plausibel und wurde für andere Coronaviren in der Maus auch schon gezeigt; dort breiteten sich die Viren transnasal über den Bulbus bis in Hippocampus, Hirnstamm und Rückenmark aus [29]. Allerdings bedeuten Riechstörungen keineswegs automatisch, dass eine ZNS-Invasion durch SARS-CoV-2 stattgefunden hat, zumal eine relevante ACE2und TPMRSS2-Expression in reifen olfaktorischen Rezeptor- neuronen, denjenigen Zellen im Riechepithel mit Verbindung nach zentral, nicht gezeigt wurde, sondern bisher in nichtneuronalen olfaktorischen Zellen [38, 39], sodass in den meisten Fällen die Riechstörung wohl eher lokal bedingt sein dürfte. Da Geschmacksstörungen auch ohne Riechstörung beschrieben wurden, wird eine direkte Schädigung der Rezeptoren durch das Virus diskutiert [40].

Alternativ zu einem axonalen Transport bliebe für die transnasale Ausbreitung eine direkte Transmission von nichtneuronalen Epithelzellen in den Liquor von olfaktorischen Neuronen in der Nähe der Lamina cribrosa. Auch andere Hirnnerven mit Verbindung zum Mund-Rachen-Raum oder den Atemorganen und das enterische Nervensystem werden als mögliche Eintrittspforten erwogen. In Abb. 1 ist die denkbare Neuroinvasion vereinfacht dargestellt.

Außer diesen primär ZNS-bezogenen pathophysiologischen Überlegungen werden zahlreiche sekundäre Auswirkungen der systemischen Erkrankung auf das Nervensystem diskutiert, darunter Zytokin-/Mediator- und metabolische Effekte der systemischen Inflammation mit Folgen wie Enzephalopathie, Delir, Anfällen [41]; zerebral hypoxische Effekte des Lun- 
genversagens; phasenabhängig entweder gerinnungsfördernde oder gerinnungshemmende Blutkonstellationen mit Folgen wie ischämischen oder hämorrhagischen Schlaganfällen; und Kreuzreaktionen bzw. andere inflammatorische Trigger von para- oder postinfektiösen Myo- und Neuropathien [36].

Ob COVID-19 nun spezifische oder eher unspezifische Auswirkungen auf das Nervensystem hat, mag zwar für Diagnostik und Therapie, die zu diesem Zeitpunkt noch völlig unklar ist, eine wichtige Rolle spielen, aber zunächst einmal werden wir zunehmend Patienten sehen, die im Zusammenhang mit der Virusinfektion neurologisch beeinträchtigt sind und optimal behandelt werden müssen. Das verlangt nach einem gesteigerten Bewusstsein für diesen Teil der Erkrankung, insbesondere bei nichtneurologischen Behandlern, und macht die Einbindung von Neurologen in die Versorgung erforderlich.

\section{Forschung zu Neuro-COVID}

Alle oben gemachten Ausführungen stehen auf einer sehr dünnen Datenbasis. Teilweise kommen die bisherigen Publikationen aus dem Prä-Print-Bereich und weisen erhebliche methodische Schwächen auf, und auch wenn die klinischen Berichte teils frappierend schnell in hochrangigen Zeitschriften wie Lancet, JAMA oder NEJM erscheinen, ist wegen eines beschleunigten Reviewverfahrens Vorsicht mit allzu weitreichenden Schlussfolgerungen geboten. Vor allem sind die retrospektiven Fallberichte, Fallserien oder Beobachtungsstudien schwerlich in der Lage, belastbar die Häufigkeit, die Bedeutung und insbesondere die Differenzierung zwischen spezifischen und unspezifischen Assoziationen von neurologischen Manifestationen bei COVID-19 zu erlauben. Dies macht es zum gegenwärtigen Zeitpunkt nahezu unmöglich, Therapieoptionen oder auch die Erkennung neurologisch nachteiliger Elemente supportiver (Intensiv-)Therapien abzuleiten.

Deshalb ist es sehr erfreulich, dass nun im Rahmen groß angelegter Registerstudien auch neurologische Aspekte Einzug finden sollen. Hier ist v. a. das europaweite, von der DGI und DZIF aufgelegte „lean european open survey for SARS-CoV-2 infected patients" (LEOSS [42]) zu nennen. Eine weitere wichtige Initiative ist eine nationale Registerstudie der DGN - Junge Neurologen, in der es u. a. um die Auswirkung von COVID-19 auf vorbestehende neurologische Erkrankungen und die neurologischen Folgen von COVID-19 geht (NeuroCoV) [43].

Einen anderen Fokus, nämlich auf die COVID-19-Patienten mit neurologischen Manifestationen und schwerem Verlauf, also Überwachungs- und Intensivpflichtigkeit, wählt das DGNI-Forschungsnetzwerk IGNITE: In der multizentrischen Studien „pooled analysis of neurologic disorders manifesting in intensive care COVID-19" (PANDEMIC) werden Patienten prospektiv auf neurologische Begleiterscheinungen gescreent und dann bei neurologischer Beteiligung diagnostisch vertieft charakterisiert [44].

Es bleibt zu hoffen, dass die Erkenntnisse aus diesen Studien nicht nur zu einer erhöhten Aufmerksamkeit für neurologische Symptome und Erkrankungen im Zusammenhang mit COVID-19, sondern auch zu wichtigen therapeutischen Beiträgen seitens der Neurologie führen. Die DGN informiert auf ihrer Homepage, z. B. im DGN Journal Club [45] kontinuierlich über die aktuelle Entwicklung dieses wichtigen Themas.
Literatur

1. Schiffmann A (2020) Coronavirus dashboard, available from https://ncov2019.live/ data. Accessed 21 Apr 2020

2. Klein BC, Busis NA (2020) COVID-19 is catalyzing the adoption of teleneurology. Neurology. https://doi.org/10.1212/wnl.0000000000009494

3. DGN (2020) https://www.dgn.org/neuronews/71-neuronews-2020/3890immuntherapien-bei-neuroimmunologischen-erkrankungen-vor-demhintergrund-der-sars-cov-2-pandemie. Accessed 06 May 2020

4. Morelli N, Rota E, Terracciano C et al (2020) The baffling case of Ischemic stroke disappearance from the casualty department in the COVID-19 era. Eur Neurol. https://doi.org/10.1159/000507666

5. Khosravani H, Rajendram P, Notario L, Chapman MG, Menon BK (2020) Protected code stroke: Hyperacute stroke management during the Coronavirus disease 2019 (COVID-19) pandemic. Stroke. https://doi.org/10.1161/strokeaha.120.029838

6. Sharma D, Rasmussen M, Han Ret al (2020) Anesthetic management of endovascular treatment of acute Ischemic stroke during COVID-19 pandemic: consensus statement from society for neuroscience in anesthesiology \& critical care (SNACC)_endorsed by society of vascular \& Interventional neurology (SVIN), society of Neurolnterventional surgery (SNIS), Neurocritical care society (NCS), and European society of minimally invasive neurological therapy (ESMINT). J Neurosurg Anesthesiol. https://doi.org/10.1097/ana.0000000000000688

7. Bersano A, Pantoni L (2020) On being a neurologist in Italy at the time of the COVID19 outbreak. Neurology. https://doi.org/10.1212/wnl.0000000000009508

8. DIVI-Empfehlung Ethik (2020) https://www.divi.de/empfehlungen/publikationen/ covid-19/1549-entscheidungen-ueber-die-zuteilung-intensivmedizinischerressourcen-im-kontext-der-covid-19-pandemie-klinisch-ethische-empfehlungen/ file. Accessed 06 May 2020

9. Kim SYH, Grady C (2020) Ethics in the time of COVID: What remains the same and what is different. Neurology. https://doi.org/10.1212/wnl.0000000000009520

10. Mao L, Jin H, Wang M et al (2020) Neurologic manifestations of hospitalized patients with Coronavirus disease 2019 in Wuhan, China. JAMA Neurol. https://doi. org/10.1001/jamaneurol.2020.1127

11. Helms J, KremerS, Merdji Het al (2020) Neurologic features in severe SARS-coV-2 infection. N Engl J Med. https://doi.org/10.1056/nejmc2008597

12. Li Y, Wang M, Zhou Y et al (2020) Acute cerebrovascular disease followinig COVID19: a single center, retrospective, observational study (manuscript draft, https://ssm. $\mathrm{com} /$ abstract $=3550025$ )

13. Zhang Y, Xiao M, Zhang Set al (2020) Coagulopathy and antiphospholipid antibodies in patients with Covid-19. NEngl J Med. https://doi.org/10.1056/ nejmc 2007575

14. Oxley TJ, Mocco J, Majidi S et al (2020) Large-vessel stroke as a presenting feature of Covid-19 in the young. N Engl J Med. https://doi.org/10.1056/nejmc2009787 15. Poyiadji N, Shahin G, Noujaim D, Stone M, Patel S, Griffith B (2020) COVID-19associated acute hemorrhagic necrotizing encephalopathy: CT and MRI features. Radiology. https://doi.org/10.1148/radiol.2020201187

16. Moriguchi T, Harii N, Goto J et al (2020) A first case of meningitis/encephalitis associated with SARS-Coronavirus-2. Int J Infect Dis 94:55-58

17. Paniz-Mondolfi A, Bryce C, Grimes Z et al (2020) Central nervous system involvement by severe acute respiratory syndrome Coronavirus-2 (SARS-CoV-2). J Med Virol. https://doi.org/10.1002/jmv.25915

18. Toscano G, Palmerini F, Ravaglia S et al (2020) Guillain-barre syndrome associated with SARS-coV-2. N Engl J Med. https://doi.org/10.1056/nejmc2009191

19. Guan WJ, Ni ZY, Hu Y et al (2020) Clinical characteristics of Coronavirus disease 2019 in China. N Engl J Med. https://doi.org/10.1001/jamanetworkopen.2020.5619

20. Zheng Y, Xu H, Yang Met al (2020) Epidemiological characteristics and clinical features of 32 critical and 67 noncritical cases of COVID-19 in Chengdu. J Clin Virol 127:104366

21. Zhou F, Yu T, Du Ret al (2020) Clinical course and risk factors for mortality of adult inpatients with COVID-19 in Wuhan, China: a retrospective cohort study. Lancet 395(10229):1054-1062

22. Chen T, Wu D, Chen H et al (2020) Clinical characteristics of 113 deceased patients with coronavirus disease 2019: retrospective study. BMJ 368:m1091 
Im Fokus

23. Dreher M, Kersten A, Bickenbach J et al (2020) The characteristics of 50 hospitalized COVID-19 patients with and without ARDS. Dtsch Arztebl 117:271-278. https:// doi. org/10.3238/arztebl.2020.0271

24. Yan CH, Faraji F, Prajapati DP, Boone CE, DeConde AS (2020) Association of chemosensory dysfunction and Covid-19 in patients presenting with influenza-like symptoms. Int Forum Allergy Rhinol. https://doi.org/10.1002/alr.22579

25. Xinhua, Bejjing hospital confirms nervous system infections by novel coronavirus, http://www.china.org.cn/china/2020-03/05/content_75777888.htm.Accessed 06 May 2020

26. Aggarwal P (1992) Endotracheal intubation following diazepam or lorazepam in status epilepticus. J Emerg Med 10(2):206-207

27. Klok FA, Kruip M, van der Meer NJM et al (2020) Incidence of thrombotic complications in critically ill ICU patients with COVID-19. Thromb Res. https://doi. org/10.1016/j.thromres.2020.04.013

28. Zhao H, Shen D, Zhou H, Liu J, Chen S (2020) Guillain-Barre syndrome associated with SARS-COV-2 infection: causality or coincidence? Lancet Neurol. https://doi.org/ 10.1016/s1474-4422(20)30109-5

29. Desforges M, Le Coupanec A, Brison E, Meessen-Pinard M, Talbot PJ (2014) Neuroinvasive and neurotropic human respiratory coronaviruses: potential neurovirulent agents in humans. Adv Exp Med Biol 807:75-96

30. Zhou P, Yang XL, Wang XG et al (2020) A pneumonia outbreak associated with a new coronavirus of probable bat origin. Nature 579(7798):270-273

31. Letko M, Marzi A, Munster V (2020) Functional assessment of cell entry and receptor usage for SARS-COV-2 and other lineage B betacoronaviruses. Nat Microbiol 5(4):562-569

32. Hoffmann M, Kleine-Weber H, Schroeder Set al (2020) SARS-coV-2 cell entry depends on ACE2 and TMPRSS2 and is blocked by a clinically proven protease inhibitor. Cell 181(2):271-280e8. https://doi.org/10.1016/j.cell.2020.02.052

33. Baig AM, Khaleeg A, Ali U, Syeda H (2020) Evidence of the COVID-19 virus targeting the CNS: tissue distribution, host-virus interaction, and proposed neurotropic mechanisms. ACS Chem Neurosci 11(7):995-998

34. Li YC, Bai WZ, Hashikawa T (2020) The neuroinvasive potential of SARS-CoV2 may play a role in the respiratory failure of COVID-19 patients. J Med Virol. https://doi. org/10.1002/jmv.25728

35. Turtle L (2020) Respiratory failure alone does not suggest central nervous system invasion by SARS-CoV-2. J Med Virol. https://doi.org/10.1002/jmv.25828

36. WU Y, XU X, Chen Z et al (2020) Nervous system involvement after infection with COVID-19 and other coronaviruses. Brain Behav Immun. https://doi.org/10.1016/j. bbi.2020.03.031

37. Hopkins C, Surda P, Kumar N (2020) Presentation of new onset anosmia during the COVID-19 pandemic. Rhinology. https://doi.org/10.1186/s40463-020-00423-8

38. Butowt R, Bilinska K (2020) SARS-coV-2: olfaction, brain infection, and the urgent need for clinical samples allowing earlier virus detection. ACS Chem Neurosci. https://doi.org/10.1021/acschemneuro.0c00172

39. Brann DH, Tsukahara T, Weinreb C, Logan DW, Datta SR (2020) Non-neuronal expression of SARS-COV-2 entry genes in the olfactory epthelium suggests mechanims underlying anosmia in COVID-19 patiens. bioRxiv preprint. https:// www.bioxriv.org/content/10.1101/2020.03.25.009084v1.avstract

40. Finsterer J, Stollberger C (2020) Causes of hypogeusia/hyposmia in SARS-CoV2 infected patients. J Med Virol. https://doi.org/10.1002/jmv.25903

41. Mehta P, MCAuley DF, Brown M, Sanchez E, Tattersall RS, Manson JJ (2020) COVID-

19: consider cytokine storm syndromes and immunosuppression. Lancet 395(10229):1033-1034

42. LEOSS (2020) https://leoss.net/. Accessed 06 May 2020

43. https://wWw.dgn.org/rubrik-themen/3958-registerstudie-zu-covid-19-derjungen-neurologen. Accessed 06 May 2020

44. https://wWw.dgni.de/forschung/ignite-initiative-klinischer-multizenter-studien. html. Accessed 06 May 2020

45. https://www.dgn.org/rubrik-themen/journal-club. Accessed 06 May 2020
Korrespondenzadresse

Prof. Dr. med. J. Bösel

Klinik für Neurologie

Klinikum Kassel

Mönchebergstr. 41-43, 34125 Kassel, Deutschland

Julian.Boesel@klinikum-kassel.de

\section{Einhaltung ethischer Richtlinien}

Interessenkonflikt. J.Bösel und P. Berlit geben an, dass kein Interessenkonflikt besteht.

Für diesen Beitrag wurden von den Autoren keine Studien an Menschen oder Tieren durchgeführt. Für die aufgeführten Studien gelten die jeweils dort angegebenen ethischen Richtlinien. 